H. NATSUME AND T. NATSUME

KODAI MATH. J.

3 (1980), 364-373

\title{
A REMARK ON THE DE RHAM MAP FOR FOLIATED MANIFOLDS
}

\author{
By Hiroko Natsume and Toshikazu Natsume
}

$\S 0$. Introduction. The notion of continuous cohomology was first introduced by R. Bott [1]. Given a space $X$ with a finer topology $X^{\prime}$, the continuous cohomology $H_{c}^{*}\left(X^{\prime} \rightarrow X\right)$ is the cohomology of the complex of singular cochains on $X^{\prime}$ which are continuous with respect to the topology of $X$. In [2] and [3], M. A. Mostow axiomatized the continuous cohomology by sheafifying $H_{c}^{*}$, and also studied smooth analogue $T_{\infty}^{*}$.

For a manifold $M$ with a foliation $F$, we denote the set $M$ with the leaf topology by $M^{F}$. Mostow defined the de Rham map of $T_{\infty}^{*}\left(M^{F} \rightarrow M\right)$ into $T_{c}^{*}\left(M^{F} \rightarrow M\right)$, a sheafified version of $H_{c}^{*}$. If we consider $(M \rightarrow M)$, then the de Rham map is the usual one. Simple examples show that the de Rham map is not necessarily surjective. Then it is natural to ask whether the de Rham map is injective or not. The present paper is a negative answer to this question.

This paper is organized as follows. In Section 1 we shall review the definitions of continuous cohomology, smooth cohomology and the de Rham map. In Section 2 we shall consider the torus $T^{2}$ foliated by lines of constant slope, and calculate the continuous and smooth cohomologies. In Section 3 it will be shown that for certain foliations on $T^{2}$ the de Rham maps are not injective.

$\S 1$. Definitions of continuous cohomology and smooth cohomology

In this section we shall give the definitions of continuous cohomology and smooth cohomology of foliated manifolds.

From now on, we assume that all manifolds are paracompact and Hausdorff spaces. Let $F$ be a foliation on a smooth manifold $M$, and let $M^{F}$ be the set $M$ topologized as the disjoint union of the leaves of $F$, each topologized as an abstract manifold (not as a subspace of $M$ ). We put $M !=\left(M^{F} \stackrel{\imath}{\rightarrow} M\right.$ ), where $i$ denotes the identity mapping. We call $M$ ! a foliated manifold. Let $\operatorname{Loc}(M !)$ denote the sheaf on $M$ consisting of germs of continuous functions $f: M \rightarrow \boldsymbol{R}$ which are constant on each leaf.

DEFINITION 1.1. The cohomology group with sheaf coefficient $H^{*}(M ; \operatorname{Loc}(M !))$ is denoted by $T_{l o c}^{*}(M !)$, and is called the continuous cohomology of a foliated manifold $M$ !.

Received July 2, 1979 
Similarly we define the smooth cohomology as follows. Let $L o c_{\infty}(M !)$ be the sheaf on $M$ consisting of germs of smooth functions $f: M \rightarrow \boldsymbol{R}$ which are constant on each leaf.

Definition 1.2. $T_{\infty}^{*}(M !)=H^{*}\left(M ; \operatorname{Loc}_{\infty}(M !)\right)$ is called the smooth cohomology of a foliated manifold $M !$.

Comparing these definitions, we see that there is a natural inclusion $\Phi$ of $\operatorname{Loc}_{\infty}(M !)$ in $\operatorname{Loc}(M !)$. Then $\Phi$ induces a homomorphism

$$
\Phi_{D R}^{q}: T_{\infty}^{q}(M !) \longrightarrow T_{l o c}^{q}(M !)
$$

for each $q$. We call the collection $\Phi_{D R}=\left\{\Phi_{D R}^{q}\right\}$ the de Rham map for $M !$.

R. Bott, A. Haefliger and M. A. Mostow defined several kinds of continuous and smooth cohomologies of spaces with two topologies ([2], [3]). But, for foliated manifolds, every definition of continuous cohomology coincides with $T_{l o c}^{*}(M$ !) (in details, see [2]). Moreover the de Rham map defined in [3] is somewhat different from the above one. But we have the following commutative diagram (the notations are the same as in [3]):

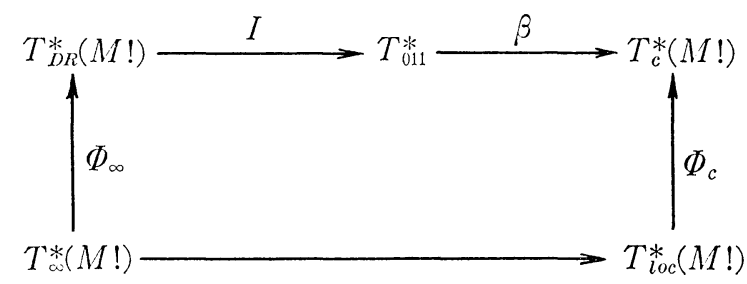

For a foliated manifold, $\Phi_{\infty}, \Phi_{c}$ and $\beta$ are isomorphisms, and in $[3] \beta^{-1} \circ I$ is called the de Rham map.

Now we give some examples. For a smooth manifold $M$ of dimension $n, M !=(M \stackrel{\imath}{\rightarrow} M)$ is a foliated manifold of codimension 0 . In this case we see that $\operatorname{Loc}_{\infty}(M !)=\operatorname{Loc}(M !)$ is equal to the constant sheaf $R$. Therefore $T_{l o c}^{*}(M !)$ $=H^{*}(M ; R)=T_{\infty}^{*}(M !)$. We give another example. Let $M_{d}$ denote $M$ with the discrete topology. Then $M_{D}=\left(M_{d} \stackrel{\imath}{\rightarrow} M\right)$ is a foliated manifold of codimension $n$. In this case we can get:

$$
\begin{aligned}
& T_{l o c}^{q}\left(M_{D}\right)=\left\{\begin{array}{cc}
C(M) & q=0 \\
0 & q>0,
\end{array}\right. \\
& T_{\propto \infty}^{q}\left(M_{D}\right)=\left\{\begin{array}{cc}
C^{\infty}(M) & q=0 \\
0 & q>0,
\end{array}\right.
\end{aligned}
$$

and the de Rham map is the natural inclusion $C^{\infty}(M) \subset C(M)$. 
2. Foliations on $T^{2}$

In this section we calculate $T_{l o c}^{*}$ and $T_{\infty}^{*}$ of certain foliations on the 2-dimensional torus $T^{2}$.

Let $X$ ! be the torus $T^{2}$ foliated by lines of constant slope. We describe this foliation more precisely. Let $\Gamma$ be the lattice of $\boldsymbol{R}^{2}$ defined by

$$
\Gamma=Z e_{1} \oplus Z e_{2},
$$

where $e_{1}=(2 \pi, 0)$ and $e_{2}=(0,2 \pi) . \quad T^{2}=S^{1} \times S^{1}$ is identified with $\boldsymbol{R}^{2} / \Gamma . \quad \boldsymbol{R}^{2}$ is foliated by lines of constant slope $\theta$. This foliation induces a foliation on $T^{2}$. Then $X !$ is $T^{2}$ with this foliation. If $\theta$ is a rational number, then all leaves are diffeomorphic to $S^{1}$. If $\theta$ is an irrational number, then each leaf, diffeomorphic to $\boldsymbol{R}$, is dense in $T^{2}$ by Kronecker's approximation theorem. The foliation $X$ ! is transversal to the fibre of the bundle $\Pi: T^{2}=S^{1} \times S^{1} \rightarrow S^{1}$, where the action of the generator 1 of $\Pi_{1}(B)=\Pi_{1}\left(S^{1}\right)=Z$ on the fibre $S^{1}$ is a rotation $r: S^{1} \rightarrow S^{1}$ defined by the formula

$$
r(z)=e^{2 \pi i \theta} z \text { for } z \in C \text { with }|z|=1 .
$$

Put $\alpha=2 \pi \theta$. If we set $\alpha=2 \pi k+\alpha^{\prime}$, where $k \in Z$ and $-\pi \leqq \alpha^{\prime} \leqq \pi$, then

$$
e^{\alpha \imath} z=e^{\alpha^{\prime} \imath} z \text {. }
$$

Therefore we may assume that $-\pi \leqq \alpha \leqq \pi$. The action of $l \in Z$ on $C\left(S^{1}\right)$ is defined by

$$
r^{*}: f \longmapsto f \circ r
$$

By definitions, $T_{\text {loc }}^{*}(X !)=H^{*}\left(T^{2} ; \operatorname{Loc}(X !)\right), T_{\infty}^{*}(X !)=H^{*}\left(T^{2} ; \operatorname{Loc}_{\infty}(X !)\right)$. The following proposition is a well-known fact of sheaf theory.

Proposition 2.1 (The Leray spectral sequence of a map). There is a spectral sequence which converges to a graded module associated to $H^{*}\left(T^{2} ; \operatorname{Loc}(X !)\right)$. The $E_{2}$-terms have the form

$$
E_{2}^{p, q}=H^{p}\left(S^{1} ; \underline{H}^{q}(\Pi ; \operatorname{Loc}(X !))\right),
$$

where $H^{q}(\Pi ; \operatorname{Loc}(X !))$ is the Leray sheaf of the map $\Pi$. Similarly, there is a spectral sequence converges to a graded module associated to $H^{*}\left(T^{2} ; \operatorname{Loc}_{\infty}(X !)\right)$. The ' $E_{2}$-terms have the form

$$
{ }^{\prime} E_{2}^{p, q}=H^{p}\left(S^{1} ; \underline{H}^{q}\left(\Pi ; \operatorname{Loc}_{\infty}(X !)\right)\right) .
$$

Moreover, the homomorphism $j: \operatorname{Loc}_{\infty}(X !) \rightarrow \operatorname{Loc}(X !)$ induces the homomorphism ' $E_{2}^{p, q} \rightarrow E_{2}^{p, q}$ which corresponds to the homomorphısm $\jmath_{*}: \underline{H}^{q}\left(\Pi ; \operatorname{Loc}_{\infty}(X !)\right)$ $\rightarrow \underline{H}^{q}(\Pi ; \operatorname{Loc}(X !))$ induced from $j$.

For the proof, see [2].

The Leray sheaf $\underline{H}^{q}(\Pi ; \operatorname{Loc}(X !))$ on the base space $S^{1}$ is generated by the presheaf ; 


$$
\begin{aligned}
U \longmapsto & H^{q}\left(\Pi^{-1} ; \operatorname{Loc}(X !) \mid \Pi^{-1}(U)\right)=T_{l o c}^{q}\left(U \times S^{1}{ }_{D}\right) \\
& =T_{l o c}^{q}\left(S^{1}{ }_{D}\right) \\
& =\left\{\begin{array}{cc}
C\left(S^{1}\right) & q=0, \\
0 & q>0 .
\end{array}\right.
\end{aligned}
$$

Since the structure group of $\Pi: T^{2} \rightarrow S^{1}$ is discrete, $\left\{C\left(S^{1}\right)\right\}=\left\{C\left(\Pi^{-1}(b)\right)\right\}_{b \in S^{1}}$ forms a local system on $S^{1}$, so that $\underline{H}^{0}$ is a locally constant sheaf. For smooth case, we get the same answer but with $C^{\infty}\left(S^{1}\right)$ in place of $C\left(S^{1}\right)$ and $T_{\infty}^{q}$ in place of $T_{l o c}^{q}$. Therefore we have

and

$$
E_{2}^{p, 0}=H^{p}\left(S^{1} ;\left\{C\left(S^{1}\right)\right\}\right),
$$

$$
{ }^{\prime} E_{2}^{p, 0}=H^{p}\left(S^{1} ;\left\{C^{\infty}\left(S^{1}\right)\right\}\right) .
$$

Then by Eilenberg's theorem ([2, Theorem 9.9]), we have

and

$$
H^{i}\left(S^{1} ;\left\{C\left(S^{1}\right)\right\}\right)=H_{E M}^{i}\left(\pi_{1}\left(S^{1}\right) ; C\left(S^{1}\right)\right),
$$

$$
H^{i}\left(S^{1} ;\left\{C^{\infty}\left(S^{1}\right)\right\}\right)=H_{E M}^{i}\left(\pi_{1}\left(S^{1}\right) ; C^{\infty}\left(S^{1}\right)\right), \quad \imath=0,1,2, \cdots .
$$

Here the action of $\pi_{1}\left(S^{1}\right)=Z$ on $C\left(S^{1}\right)$ is the above one. As in [2, p. 98] we have

Similarly,

$$
\begin{aligned}
& T_{l o c}^{0}(X !)=H^{0}\left(Z ; C\left(S^{1}\right)\right)=\left\{f \in C\left(S^{1}\right) ; f-f \circ r=0\right\} \\
& T_{l o c}^{1}(X !)=H^{1}\left(Z ; C\left(S^{1}\right)\right)=C\left(S^{1}\right) /\left\{f-f \circ r \mid f \in C\left(S^{1}\right)\right\} .
\end{aligned}
$$

$$
\begin{aligned}
& T_{\infty}^{0}(X !)=\left\{f \in C^{\infty}\left(S^{1}\right) ; f-f \circ r=0\right\}, \\
& T_{\infty}^{1}(X !)=C^{\infty}\left(S^{1}\right) /\left\{f-f \circ r ; f \in C^{\infty}\left(S^{1}\right)\right\} .
\end{aligned}
$$

Moreover the de Rham map $T_{\infty}^{i}(X !) \rightarrow T_{l o c}^{\imath}(X !)(\imath=0,1)$ coincides with the map induced from the natural inclusion

$$
\jmath: C^{\infty}\left(S^{1}\right) \longrightarrow C\left(S^{1}\right)
$$

Therefore, putting $\Psi: C^{\infty}\left(S^{1}\right) /\{f-f \circ r\} \rightarrow C^{0}\left(S^{1}\right) /\{f-f \circ r\}$ be the map induced from $\jmath$, we obtain a commutative diagram;

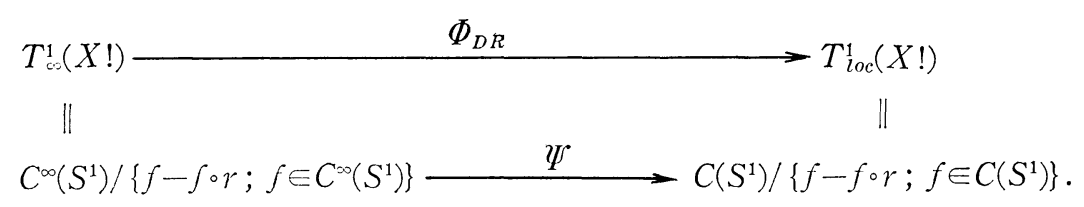

We investigate the map $\Psi$.

A real number $\alpha / 2 \pi$ defines a rotation $r$ of $S^{1}$ by the formula; 


$$
r(x)=e^{\alpha \imath} \cdot x,
$$

where $\cdot$ denotes the multiplication in $\boldsymbol{C}$.

Now we can formulate our main results as follows.

THEOREM 2.1. (I) If $\alpha / 2 \pi$ is a rational number, there exist isomorphisms

and

$$
\phi_{\infty}: C^{\infty}\left(S^{1}\right) \longrightarrow C^{\infty}\left(S^{1}\right) /\left\{f-f \circ r: f \in C^{\infty}\left(S^{1}\right)\right\}
$$

$$
\phi_{c}: C\left(S^{1}\right) \longrightarrow C\left(S^{1}\right) /\left\{f-f \circ r ; f \in C\left(S^{1}\right)\right\}
$$

such that the following diagram is commutative;

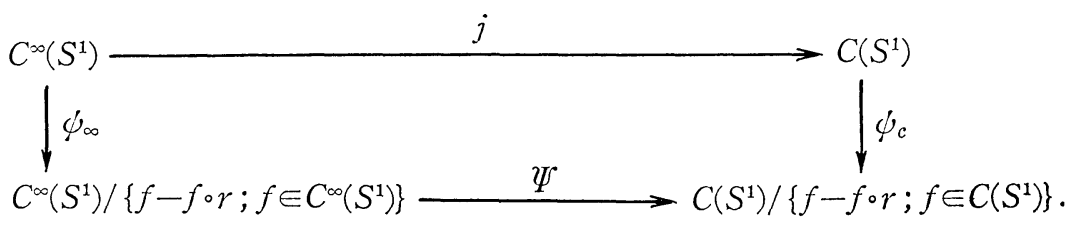

(II) If $\alpha / 2 \pi$ is not a Liouville number, and if $f \in C^{\infty}\left(S^{1}\right)$ is written in the form $f=g-g \circ r$ for some $g \in C\left(S^{1}\right)$, then $g$ is smooth.

(III) If $\alpha / 2 \pi$ is a Liouville number, then for any positive integer $k$, there exists a function $g$ of class $C^{k}$ but not of class $C^{k+1}$ defined on $S^{1}$ such that $f=g-g \circ r$ is smooth.

COROllary to THEOREM 2.1. If $\alpha / 2 \pi$ is a Liouville number, then the de Rham map $\Phi_{D R}: T_{\infty}^{1}(X !) \rightarrow T_{l o c}^{1}(X !)$ is not injective.

Remark. $\alpha / 2 \pi$ is not a Liouville number if and only if there exist some integer $p>0$ and some real number $\varepsilon>0$ such that

$$
|\alpha m+2 \pi n|>\varepsilon(|m|+|n|)^{-p}
$$

for any pair of integers $(m, n) \neq(0,0)$.

\section{Proof of Theorem}

In this section we give the proofs of our main results.

\subsection{Proof of (I)}

For the sake of simplicity, we put $I^{\infty}(r)=\left\{f-f \circ r ; f \in C^{\infty}\left(S^{1}\right)\right\}$ and $I(r)$ $=\left\{f-f \circ r ; f \in C\left(S^{1}\right)\right\}$. If we write $\alpha / 2 \pi=q / p$ with $(p, q)=1, p>0$, then $r(x)$ $=e^{\alpha 2} \cdot x=e^{2 \pi \imath \cdot q / p} \cdot x$. It is obvious that $r^{p}(=p$-times iteration of $r)$ is equal to identity mapping. We can identify $C\left(S^{1}\right)$ with the vector space $P^{0}$ consisting of continuous functions on $\boldsymbol{R}$ which are periodic of period $2 \pi$. Through this identification, we identify $C^{\infty}\left(S^{1}\right)$ with $P$ consisting of smooth functions contained in $P^{0}$. Then the action of $r$ on $P^{0}$ can be written as follows; 


$$
\left(r^{*} f\right)(y)=f(\alpha+y) .
$$

It is clear that $r^{*}(P) \subset P$. We put $\tilde{I}^{\infty}(r)=\{f-f \circ r ; f \in P\}$ and $\tilde{I}(r)=\{g-g \circ r$; $\left.g \in P^{0}\right\} . \quad I^{\infty}(r)$ and $I(r)$ are identified with $\tilde{I}^{\infty}(r)$ and $\tilde{I}(r)$ respectively.

Now we define $\tilde{\psi}_{\infty}: P \rightarrow P / \tilde{I}^{\infty}(r)$ as follows;

$$
\tilde{\psi}_{\infty}(f)=\text { the class }[\tilde{f}] \text { of } \tilde{f} \text {, }
$$

where $\tilde{f} \in P$ is defined by $\tilde{f}(y)=f(p y)$. Then, by easy calculations, we can show that $\tilde{\phi}_{\infty}$ is an isomorphism of $C^{\infty}\left(S^{1}\right)$ onto $C^{\infty}\left(S^{1}\right) / I^{\infty}(r)$ and that the following diagram is commutative ;

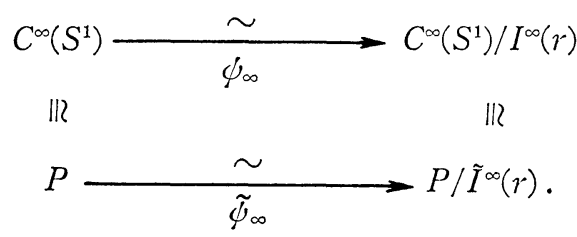

Similarly, we can obtain an isomorphism

$$
\psi_{c}: C\left(S^{1}\right) \longrightarrow C\left(S^{1}\right) / I(r) .
$$

The maps $\psi_{\infty}$ and $\psi_{c}$ are essentially the change of period, hence it is obvious that the diagram in the assertion (I) is commutative.

Thus the proof is completed.

\subsection{Proofs of (II) and (III)}

For the proofs, we may assume that all functions have values in $\boldsymbol{C}$.

Let $L^{2}$ be the space of complex valued functions defined on $\boldsymbol{R}$ which are periodic of period $2 \pi$ and have the property;

$$
\int_{-\pi}^{\pi}|f(x)|^{2} d x<\infty
$$

Then $L^{2}$ is a Hilbert space with the inner product:

$$
(f, g)=\frac{1}{2 \pi} \int_{-\pi}^{\pi} f(x) \overline{g(x)} d x, \quad \text { for } f, g \in L^{2} .
$$

Put $P_{C}=P \times{ }_{R} C$, then $P_{C}$ is a dense subset of $L^{2}$. It is a well-known fact of classical Fourier analysis that $\left\{e^{2 n x}\right\}_{n \in Z}$ form a complete orthonormal base for $L^{2}$.

Remind that for each non-negative integer $k$, the Sobolev space $H_{k}$ is the subspace of $L^{2}$ defined by

$$
H_{k}=\left\{u \in L^{2} ; \sum_{n=-\infty}^{\infty}\left(1+n^{2}\right)^{k}\left|u_{n}\right|^{2}<\infty\right\},
$$

where $\left\{u_{n}\right\}$ is the Fourier coefficient of $u$ defined by the formula; 


$$
u_{n}=\frac{1}{2 \pi} \int_{-\pi}^{\pi} u(x) e^{-\imath n x} d x
$$

If $u \in L^{2}$ is of class $C^{k}$, then clearly $u \in H_{k}$. Thus $P_{C} \subset H_{k}$ for each $k$.

The following is a fundamental lemma due to Sobolev.

LEMMA 3. 1. If $u \in H_{k}$ where $k \geqq m+1$, then $\sum n^{l} u_{n} e^{\imath n x}$ converges unformly for $l \leqq m$. Thus each $u \in H_{k}$ with $k \geqq m+1$ is equal, almost everywhere, to a function $\sum u_{n} e^{\imath n x}$ of class $C^{m}$.

For the proof, see [4, p. 237].

Now we show the assertion(II).

Proposition 3.3. Let $-\frac{1}{2}<\frac{\alpha}{2 \pi}<\frac{1}{2}$ be not a Liouville number. Then for any $f \in P_{C}$ such that

$$
\int_{-\pi}^{\pi} f(x) d x=0
$$

there exists some $g \in P_{C}$ such that $f=g-g \circ r$.

Proof. Since $\left\{e^{2 n x}\right\}$ is a complete orthonormal base, we can write as $f(x)=\sum_{n=-\infty}^{\infty} C_{n} e^{\imath n x}$, and this series converges uniformly. The condition $\int_{-\pi}^{\pi} f(x) d x$ asserts that $C_{0}=0$. We put

$$
g(x)=\sum_{n \neq 0} \frac{C_{n}}{1-e^{2 n \alpha}} e^{\imath n x} .
$$

We shall prove that for any non-negative integer $k, g \in H_{k}$. In order to see that, we must show that

$$
\sum n^{2 k}\left|\frac{C_{n}}{1-e^{2 n \alpha}}\right|^{2}=\frac{1}{4} \sum n^{2 k} \frac{\left|C_{n}\right|^{2}}{\sin ^{2}\left(\frac{n \alpha}{2}\right)}<\infty .
$$

For each integer $n$, there exists some integer $k$ such that

$$
-1 / 2<n \alpha / 2 \pi+k<1 / 2 \text {. }
$$

Then $-\frac{\pi}{2}<\frac{n \alpha}{2}+\pi k<\frac{\pi}{2}$, and $\sin ^{2}(n \alpha / 2)=\sin ^{2}(n \alpha / 2+k \pi)$. Since $-\frac{1}{2}<\frac{\alpha}{2 \pi}<\frac{1}{2}$, we have $|m| \geqq|k|$.

It is easy to see that the function $y=\sin x-\left(x-\frac{x^{3}}{6}\right)$ is non-negative on $[0, \pi / 2]$. Therefore 


$$
\sin ^{2} x \geqq\left(x-\frac{x^{3}}{6}\right)^{2} \geqq \frac{x^{6}}{36}, \quad \text { on } \quad[-\pi / 2, \pi / 2] \text {. }
$$

Hence, for some constant $C^{\prime}>0$

$$
\sin ^{2}\left(\frac{n \alpha}{2}\right) \geqq C^{\prime}|n \alpha+2 k \pi|^{6} .
$$

Since $\alpha / 2 \pi$ is not a Liouville number, there exist $\varepsilon>0$ and an integer $p \geqq 0$ such that

$$
|n \alpha+2 k \pi| \geqq \varepsilon(|n|+|k|)^{-p}
$$

for any pair of integers $(n, k) \neq(0,0)$. Therefore there exists some constant $C>0$ such that

$$
\sin ^{2}\left(\frac{n \alpha}{2}\right) \geqq C(|n|+|k|)^{-6 p} \geqq C(2|n|)^{-6 p},
$$

because $|n| \geqq|k|$. Since $f$ is smooth, $\sum n^{21}\left|C_{n}\right|^{2}<\infty$ for any $l$. Therefore, for some constant $C_{1}^{\prime}$ we have

$$
\sum_{n \neq 0} \frac{\left|C_{n}\right|^{2}}{\sin ^{2}\left(\frac{n \alpha}{2}\right)} n^{2 k} \leqq C_{1}^{\prime} \Sigma\left|C_{n}\right|^{2} n^{2 k} n^{6 p}<\infty .
$$

Thus $g \in H_{k}$ for each $k$, hence $g$ is smooth by Sobolev lemma. In particular,

$$
\sum_{n \neq 0} \frac{C_{n}}{1-e^{\imath n \alpha}} e^{\imath n x}
$$

converges uniformly. We can see that

$$
(g \circ r)(x)=\sum_{n \neq 0} \frac{C_{n}}{1-e^{\imath n \alpha}} e^{\imath n x} e^{\imath n \alpha} .
$$

Then

$$
\begin{aligned}
(g-g \circ r)(x) & =\sum_{n \neq 0} \frac{C_{n}}{1-e^{2 n \alpha}} e^{\imath n x}-\sum_{n \neq 0} \frac{C_{n}}{1-e^{2 n \alpha}} e^{2 n x} e^{2 n \alpha} \\
& =\sum_{n \neq 0} C_{n} e^{2 n x} \\
& =f(x) .
\end{aligned}
$$

Thus Proposition 3.3 is proved.

Remark 1. Let $p$ be the least non-negative integer such that for some $\varepsilon>0$,

$$
|n \alpha+2 k \pi| \geqq(|n|+|k|)^{-p}
$$

for any pair of integers $(n, k) \neq(0,0)$. Then the above arguments shows that if 
$f$ is of class $C^{m}$ with $m>3 p$, then there exists $g$ of class $C^{m-3 p-1}$ such that $f=g-g \circ r$.

Remark 2. It is a well-known fact that for irrational $\alpha / 2 \pi$, the set $\left\{r^{n}\left(x_{0}\right)\right\}_{n \in Z}$ is dense in $S^{1}$ for any point $x_{0}$. From this, it follows that if, for some continuous $g, g-g \circ r$ is equal to zero, then $g$ is a constant function. This shows that the way of decomposition $f=g-g \circ r$ is unique up to constant difference.

From Proposition 3.3 and the above Remark 2, it follows that if a smooth function $f$ is written in the form $f=g-g \circ r$, for some continuous function $g$, then $g$ itself is smooth. Thus the assertion (III) is proved.

Let now $\alpha / 2 \pi$ be a Liouville number. From the definition, it follows that for any integers $p \geqq 0$ and $N_{0} \geqq 0$, there exists a pair of integers $\left(n_{p}, k_{p}\right) \neq(0,0)$ such that

$$
\begin{aligned}
& \text { 1) }\left|n_{p}\right|>N_{0} \\
& \text { 2) }\left|n_{p} \alpha+2 k_{p} \pi\right|<\left(\left|n_{p}\right|+\left|k_{p}\right|\right)^{-p} .
\end{aligned}
$$

We may assume that $n_{p} \geqq 0$. We can take inductively $n_{p}(p=0,1, \cdots)$ such that

$$
\begin{aligned}
& \text { 3) } 0<n_{0}<n_{1}<\cdots \uparrow \infty, \\
& \text { 4) }\left|n_{p} \alpha+2 k_{p} \pi\right|<n_{p}^{-p} \quad \text { for some } k_{p} .
\end{aligned}
$$

Moreover we can take as

$$
\text { 5) } n_{p}>2^{p}(p=0,1, \cdots)
$$

For fixed $k$, we put

$$
C_{n}=\left\{\begin{array}{cl}
\frac{\sin \left(n_{p} \alpha / 2\right)}{n_{p}^{k+1}} & \text { if } n=n_{p}, \\
0 & \text { otherwise. }
\end{array}\right.
$$

Then

$$
\left|C_{n_{p}}\right|^{2} \leqq \frac{\sin ^{2}\left(n_{p} \alpha / 2\right)}{n_{p}^{2(k+1)}} \leqq \frac{1}{4} \cdot \frac{\left|n_{p} \alpha+2 k_{p} \pi\right|^{2}}{n_{p}^{2(k+1)}}<\frac{1}{4} n_{p}^{-p-2(k+1)} .
$$

Hence, for any $m \geqq 0$,

$$
\begin{aligned}
\sum n^{2 m}\left|C_{n}\right|^{2} & \leqq \frac{1}{4} \sum_{p \geqq 0} n_{p}^{2 m-p-2(k+1)} \\
& =C+\frac{1}{4} \sum_{p>2 m-2(k+1)} \frac{1}{n_{p}^{p+2(k+1)-2 m}}<\infty,
\end{aligned}
$$

because of 5 ). Therefore, for each $m \geqq 0$, the function

$$
f(x)=\Sigma C_{n} e^{2 n x}
$$

is contained in $H_{m}$, hence $f$ is smooth by Sobolev lemma. 
Put

$$
g(x)=\sum_{n \neq 0} \frac{C_{n}}{1-e^{\imath n \alpha}} e^{\imath n x} .
$$

For any $0 \leqq m \leqq k$,

$$
\begin{gathered}
\sum\left|\frac{n^{m} C_{n}}{1-e^{2 n \alpha}}\right|=\sum \frac{\left|C_{n}\right| n^{m}}{4|\sin (n \alpha / 2)|}=\frac{1}{4} \sum_{p=0}^{\infty} n_{p}^{m} n_{p}^{-(k+1)} \\
=\frac{1}{4} \sum_{p=0}^{\infty} n_{p}^{m-k-1}<\frac{1}{4} \sum_{p=0}^{\infty} 2^{p(m-k-1)}<\infty,
\end{gathered}
$$

because $m-k-1<0$. Therefore, for $0 \leqq m \leqq k$, the series

$$
\sum_{n \neq 0} n^{m} \frac{C_{n}}{1-e^{2 n \alpha}} e^{2 n x}
$$

converges uniformly. Hence $g$ is of class $C^{k}$. But

$$
\begin{gathered}
\sum n^{2(k+1)}\left|\frac{C_{n}}{1-e^{2 n \alpha}}\right|^{2}=\frac{1}{4} \sum n^{2(k+1)} \frac{\left|C_{n}\right|^{2}}{\sin ^{2}(n \alpha / 2)} \\
=\frac{1}{4} \sum_{p} n_{p}^{2(k+1)} \frac{1}{n_{p}^{2(k+1)}}=\frac{1}{4} \sum_{p} 1=\infty .
\end{gathered}
$$

Therefore $g$ is not an element of $H_{k+1}$, hence is not of class $C^{k+1}$. It is clear that $f=g-g \circ r$.

Thus the proof of the assertion (III) is completed.

The assertion (III) and Remark 2 claim that if $\alpha / 2 \pi$ is a Liouville number, then the de Rham map

is not injective.

$$
\Phi_{D R}: T_{\infty}^{1}(X !) \longrightarrow T_{l o c}^{1}(X !)
$$

Thus Corollary to Theorem 2.1 is proved.

\section{REFERENCES}

[1] R. Bотт, Some remarks on continuous cohomology, Proc. Internat. Congress on Manifolds (Tokyo, 1973), 1975, pp. 161-170.

[2] M.A. Mostow, Continuous cohomology of spaces with two topologies, Mem. Amer. Math. Soc. No. 175 (1976).

[3] M.A. Mostow, Variations, characteristic classes, and the obstruction to mapping smooth to continuous cohomology, Trans. Amer. Math. Soc. 240 (1978), pp. 163 182.

[4] F.W. WARner, Foundations of differentiable manifolds and Lie groups, Scott, Foresman, and Co., Glenview, Ill., 1971.

Department of Mathematics

FACULTY OF SCIENCE

Tokyo Institute of Technology

Department of Mathematics

Faculty of Science

SaITAMA University 